\title{
Benign, Self-Resolving Subcutaneous Emphysema and Abdominal Crepitus after Open Gynecologic Surgery
}

\author{
Conway $\mathrm{Xu}, \mathrm{MD}^{1^{*}}$, John C Elkas, $M D^{2}$ and Chad A Hamilton, $M D^{3}$ \\ ${ }^{1}$ Department of Obstetrics and Gynecology, Inova Fairfax Hospital, Virginia, USA \\ ${ }^{2}$ Mid Atlantic Gynecologic Oncology and Pelvic Surgery Associates, Virginia, USA \\ ${ }^{3}$ Division of Gynecologic Oncology, Ochsner Medical Center and Cancer Institute, Louisiana, USA
}

*Corresponding author: Conway Xu, MD, Department of Obstetrics and Gynecology, $2^{\text {nd }}$ Floor South Tower, Inova Fairfax Hospital, 3300 Gallows Road, Falls Church, Virginia, 22042, USA, Tel: 703-776-2745

\begin{abstract}
Abdominal crepitus and associated subcutaneous emphysema on imaging following open gynecologic surgery typically signals life-threatening complications including perforated viscus and necrotizing fasciitis, which require prompt intervention. This case is the second to date after open gynecologic surgery where, after extensive workup, significant subcutaneous emphysema of uncertain etiology was diagnosed with a benign and self-resolving course without any long term sequalae.
\end{abstract}

\section{Keywords}

Subcutaneous emphysema, Crepitus, Abdomen, Myomectomy, Laparotomy, Benign

\section{Introduction}

Subcutaneous emphysema occurs when gas becomes trapped under the skin. Crepitus, the characteristic crackling feel, is the associated physical exam finding. Though common following laparoscopic surgery, abdominal or chest wall crepitus after open surgery should raise immediate concern for traumatic injury or infection [1]. Possible traumatic injuries include spontaneous pneumothorax and perforated viscus with subsequent migration of air through the subcutaneous space, both of which have been extensively reported in the literature [2-8]. The most common infectious etiology is necrotizing fasciitis. To our knowledge, there has only been one previous report of benign postoperative subcutaneous emphysema following open gynecologic surgery [9]. In this prior study, the patient underwent exploratory laparotomy and total abdominal hysterectomy for a large fibroid uterus. She developed massive subcutaneous emphysema during her postoperative course which was self-limited without identifiable etiology. In this report, we present a second case of benign, self-resolving abdominal crepitus and subcutaneous emphysema following open gynecologic surgery.

\section{Case Description}

A 34-year-old Black woman who desired future fertility presented for myomectomy due to symptomatic uterine fibroids manifested by urinary frequency, constipation, and pelvic pressure. She presented with anemia while all other medical and surgical history were benign and ultimately noncontributory to her case. On pelvic exam, her surgeon noted a 14-week size uterus. Her pelvic ultrasound demonstrated a $10.4 \times 6 \times 6.9 \mathrm{~cm}$ uterus with multiple fibroids with the largest measuring $7.3 \times 5.7 \times 6.2 \mathrm{~cm}$. Magnetic resonance imaging confirmed the size and location of her fibroids.

The surgeon performed an abdominal myomectomy via a low transverse incision. Intraoperative findings were consistent with her preoperative imaging. The fibroids were removed without disrupting the endometrial cavity. The myometrial defects were closed in layers and the fascia and skin were closed in standard fashion, all with delayed absorbable suture. Estimated blood loss was $500 \mathrm{~mL}$ and the surgery was uncompli-

\footnotetext{
Citation: Xu C, Elkas JC, Hamilton CA (2021) Benign, Self-Resolving Subcutaneous Emphysema and Abdominal Crepitus after Open Gynecologic Surgery. Obstet Gynecol Cases Rev 8:193. doi. org/10.23937/2377-9004/1410193

Accepted: February 16, 2021: Published: February 18, 2021

Copyright: (c) 2021 Xu C, et al. This is an open-access article distributed under the terms of the Creative Commons Attribution License, which permits unrestricted use, distribution, and reproduction in any medium, provided the original author and source are credited.
} 
cated.

Postoperatively, the patient was symptomatically anemic and she was transfused 2 units of packed red blood cells with resolution of her symptoms. Her physical exam was notable for significant crepitus starting on postoperative day 2 . The crepitus extended from her mons pubis to her xiphoid process and laterally to her flanks. The patient only reported mild bowel gas pain. Her abdominal exam was appropriately tender to palpation along her incision and otherwise soft without rebound or guarding. Her labs all trended normal with stabilization of her hemoglobin after transfusion. By postoperative day 3 , the patient's subcutaneous em- physema persisted and though she passed flatus, she still had minimal oral intake. Computed tomography (CT) scan of the chest, abdomen, and pelvis confirmed subcutaneous air along the anterior abdominal and pelvic wall, but no pneumothorax, underlying abscess, or obvious bowel perforation (Figure 1, Figure 2 and Figure 3 , left). Delayed CT imaging repeated several hours later showed progression of oral contrast through intact bowels, helping to rule out an underlying perforated viscus. The patient's oral intake normalized and meeting all postoperative milestones, she was discharged on postoperative day 5 . She was readmitted on postoperative day 7 with abdominal pain and distension. Her ab-
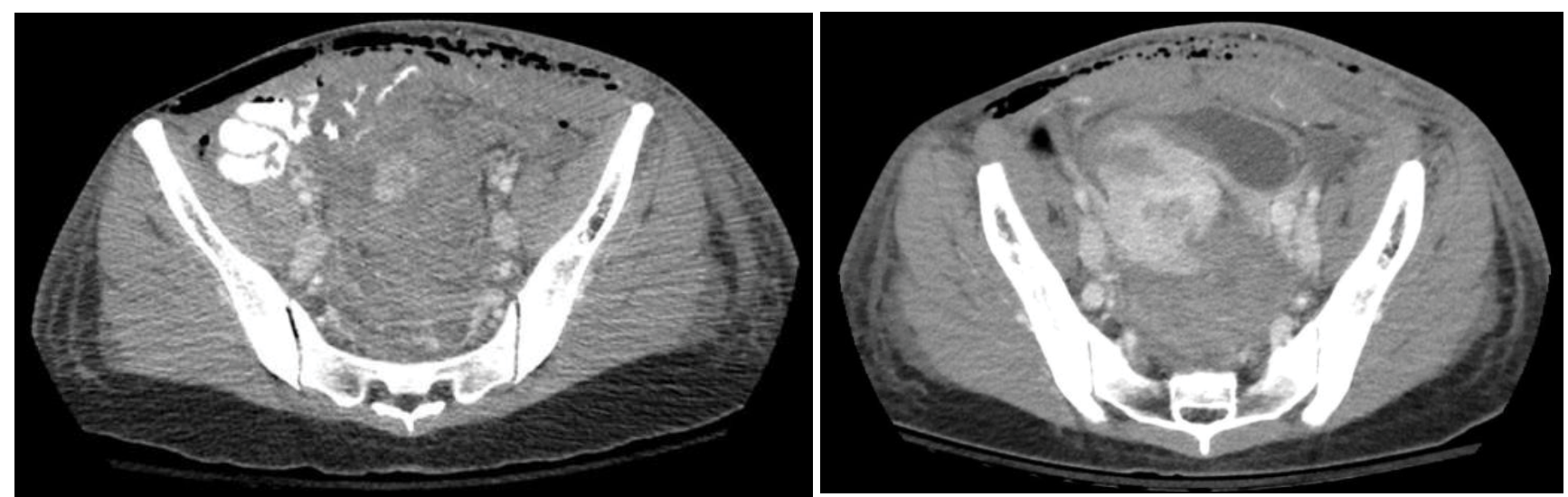

Figure 1: Subcutaneous emphysema (axial view) on post-operative day 4 (left) vs. post-operative day 7 (right).
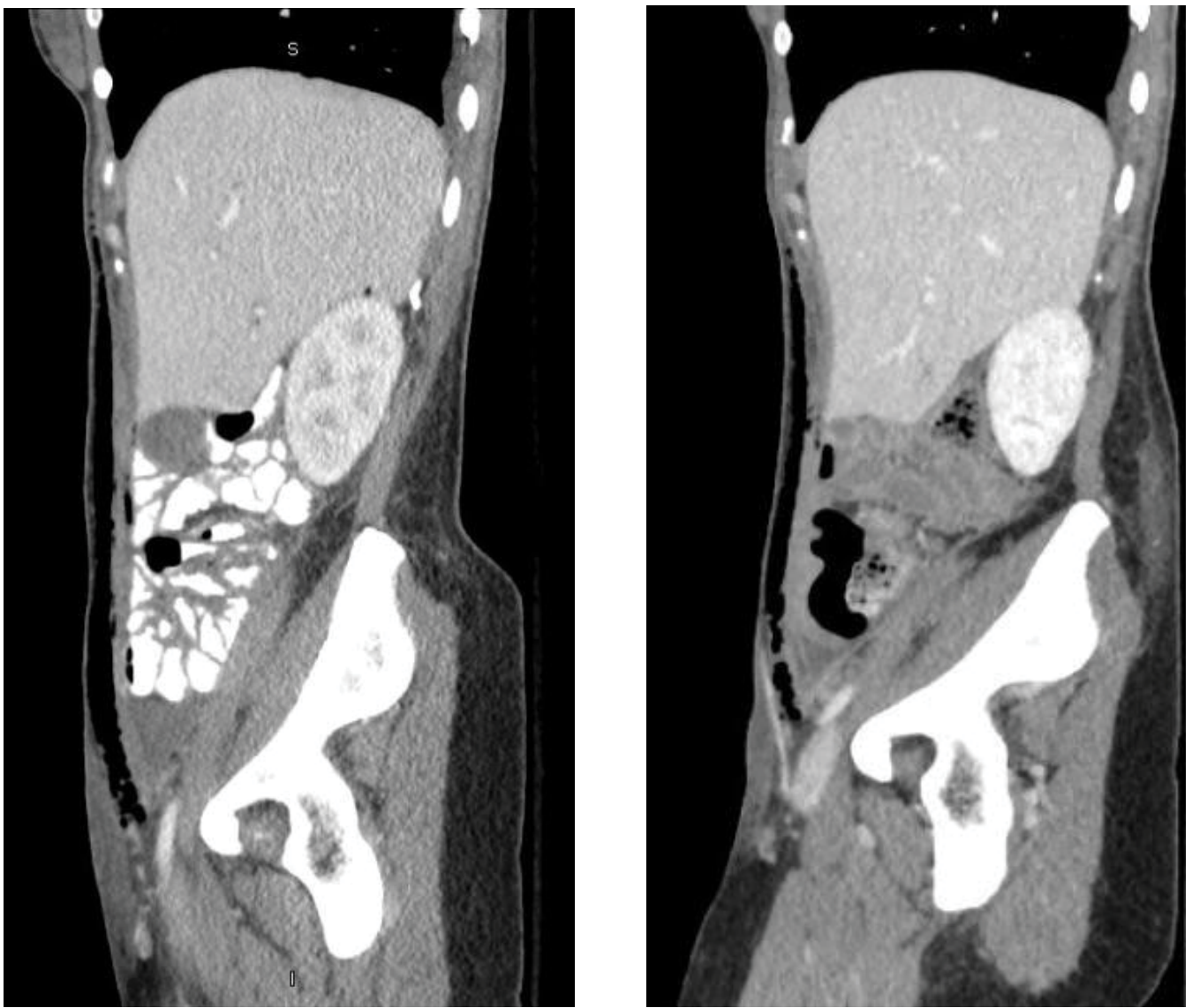

Figure 2: Subcutaneous emphysema (sagittal view) on post-operative day 4 (left) vs. post-operative day 7 (right). 

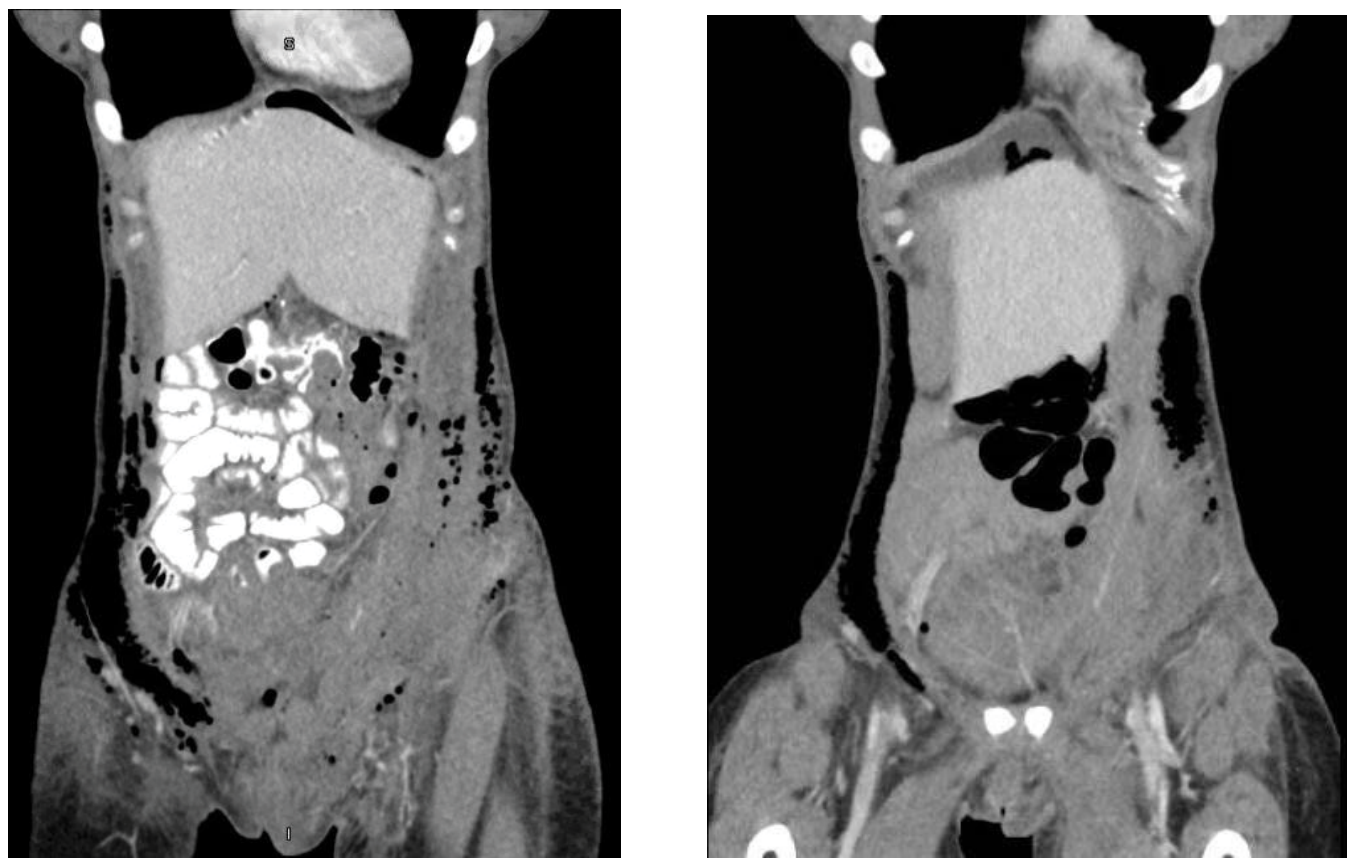

Figure 3: Subcutaneous emphysema (coronal view) on post-operative day 4 (left) vs. post-operative day 7 (right).

dominal crepitus had significantly decreased and repeat $\mathrm{CT}$ of the abdomen and pelvis demonstrated interval improvement of her subcutaneous emphysema (Figure 1 , Figure 2 and Figure 3, right). She was discharged the next morning significantly improved with conservative management.

At the patient's two-week postoperative visit, she was off of all pain medication and recovering well. Her abdominal exam was normal without crepitus. The remainder of her postoperative course was uncomplicated.

\section{Conclusion}

Postoperative subcutaneous emphysema and associated abdominal crepitus is a concerning finding with differential diagnoses including perforated viscus or necrotizing fasciitis. After ruling out these life-threatening possibilities, this case and the prior reported case provide reassurance that this may be a benign self-limiting finding of undetermined etiology after open gynecologic surgery [9]. Foote, et al. postulated that the subcutaneous emphysema likely resulted from intra-abdominal free air traveling along the extensively dissected retroperitoneum; however, the retroperitoneum was not entered during our patient's uncomplicated surgery, making this explanation less likely [9]. Alternatively, Kassir, et al. suggested that a pressure gradient between the peritoneal cavity and surrounding structures may cause a rupture of the anterior abdominal wall, then allowing intra-abdominal air to permeate along subcutaneous tissue planes [8]. While no significant defect of the anterior abdominal wall was noted on CT imaging in our patient, it is possible that micro-perforations developed or persisted immediately after surgery to facilitate this benign, self-resolving finding.

\section{Conflict of Interest}

None.

\section{Sources of Support}

None.

\section{Statement of Equal Authors' Contribution}

All authors contributed equally to this manuscript.

\section{References}

1. Ferguson CM (1990) Inspection, auscultation, palpation, and percussion of the abdomen. In: clinical methods: The history, physical and laboratory examinations. ( $3^{\text {rd }}$ edn), Butterworths, Boston.

2. Fiss JR TW, Cigtay OS, Miele AJ, Twigg HL (1975) Perforated viscus presenting with gas in the soft tissues (subcutaneous emphysema). Am J Roentgenol Radium Ther Nucl Med 125: 226-233.

3. Ota H, Fujita S, Nakamura T, Tanaka S, Tono T, et al. (2003) Pneumoretroperitoneum, pneumomediastinum, pneumopericardium, and subcutaneous emphysema complicating sigmoidoscopy: Report of a case. Surgery Today 33: 305-308.

4. Tomasoa NB, Ultee JM, Vrouenraets BC (2008) Retroperitoneal abscess and extensive subcutaneous emphysema in perforated appendicitis: A case report. Acta chirurgica Belgica 108: 457-459.

5. Ignjatovic M, Jovic J (2009) Tension pneumothorax, pneumoretroperitoneum, and subcutaneous emphysema after colonoscopic polypectomy: A case report and review of the literature. Langenbecks Arch Surg 394: 185-189.

6. Agaba EA, Kandel AR, Agaba PO, Wong LS (2010) Subcutaneous emphysema, muscular necrosis, and necrotizing fasciitis: An unusual presentation of perforated sigmoid diverticulitis. South Med J 103: 350.

7. Kipple JC (2010) Bilateral tension pneumothoraces and subcutaneous emphysema following colonoscopic polyp- 
ectomy: A case report and discussion of anesthesia considerations. AANA J.

8. Kassir R, Abboud K, Dubois J, Baccot S, Debs T, et al (2014) Perforated diverticulitis of the sigmoid colon causing a subcutaneous emphysema. International Journal of Surgery Case Reports.
9. Foote JR, Kohler MF, Young-Pierce J (2014) Case report of massive subcutaneous emphysema: A benign postoperative finding. Obstet Gynecol Cases Rev. 